\title{
INSIGHTS
}

\section{Family reflections: our family's journey with Wolfram syndrome}

\author{
Tracy Lynch ${ }^{1}$ \\ Pediatric Research (2020) 87:608; https://doi.org/10.1038/s41390-019-0661-8
}

Wolfram syndrome is an autosomal recessive inherited genetic ultra-rare progressive neurodegenerative condition with four main features-diabetes insipidus, diabetes mellitus, optic atrophy and deafness. No two people seem to be affected in the same way. WS can also cause anxiety, aggression, depression, loss of gag reflex/ choking, respiratory issues, balance and memory loss, sleep apnoea and in extreme cases scoliosis. Being on the autistic spectrum is also seeming to be mentioned more by parents of those affected. Generally the first sign is early-onset type 1 diabetes, followed by colour blindness and worsening of vision by early teens. Diabetes insipidus and hearing issues follow from the mid-teens. The average life expectancy is 30-40 years of age; but this can vary depending on an affected person's health and severity of the syndrome. WS affects 1 in 770,000 people in the United Kingdom. Many doctors have and never will see a person with WS.

My daughter was diagnosed with WS at the age of 8 following 3 years of investigations. Her development of the condition seems to have started when she was about 2.5 years old with coughing attacks, followed by vision issues diagnosed when she was 5 . At the age of 6 she was diagnosed with type 1 diabetes and a neurogenic bladder. At the age of 10 she had the second of two severe choking attacks in a year, which resulted in being on a ventilator for 3 weeks with three failed attempts at coming off, which resulted in a 'temporary' tracheostomy being carried out. Seven years later this is still in place and looks likely to remain so. At the age of 12 she was diagnosed with scoliosis, and over the last 3 years, she has developed short-term memory loss with her balance deteriorating so much that a wheelchair is required if we are out for any length of time or walking any distance. Around the age of 6 she had the first of several sleep studies that showed she had central obstructive sleep apnoea. She would desaturate for up to 13 seconds at a time, up to 20 times in an hour, but never requiring any medical intervention. We have been told that she has an extreme form of WS as everything has happened within the first 10 years of her life.

My daughter has not been able to live her life as she should. She never really had a normal childhood as time was spent out of school at hospital appointments and she stopped being invited to friend's houses and birthday parties from about the age of 6 . Once she had the tracheostomy, life changed again as she required an adult to be nearby for assistance at any time that it was required. Unlike her peers she cannot go out and get a part-time job; she cannot drive a car; she cannot go out to a bar. Wherever she goes she has to have either one of us parents or a personal assistant go with her.

Over the years she has received the necessary treatment and medication for the different parts of the condition she is affected by. She has five insulin injections a day, at least three tablets a day, one twice a day, and is now also on a ventilator at night along with any additional medications as required. In 2016, she was admitted to hospital seven times with either an upper respiratory tract infection or pneumonia. This is when the ventilator was first introduced and was used as and when required. In 2017, this became a permanent part of night time with azithromycin prescribed as a prophylactic preventive antibiotic for over the winter months. So far this has helped. My daughter is seen at the annual MDT Clinic in Birmingham, UK, and then has other medical appointments throughout the year at our local hospital and hospitals in London, about 60 miles away.

Since 2007 when our hospital journey began we have seen almost every department in the hospital. She has had blood tests and lumbar punctures; she has been seen in ophthalmology, audiology, urology, neurology, MRI and radiology, genetics, metabolics, psychology, cardiology, neurophysiology, ENT, intensive care, endocrinology, orthopaedics, respiratory and long-term ventilation.

We know that a 'sticking plaster' treatment is being trialled to slow down or even halt the progression, but we would like to see more researchers working together, sharing more information to find an actual cure. We know that there is lot of research going on around the world for different possibilities of what could be a cure. The 'sticking plaster' gives those affected and their families hope that they might live longer than the expected average age of 30-40 years. The hardest part of the condition for those affected is the loss of vision and I know this is what every person with WS would like to see 'cured' first. Everyone can cope with the other parts of the syndrome with medication, but not being able to see is the hardest thing, no longer being able to do things easily for yourself when once you could is tough on them as well as the whole family. As a parent, watching your child's health and their condition deteriorate is really tough. The bladder is the second choice for being cured on the list.

As a parent, I would ask researchers and doctors working on this condition to listen to the individuals and families affected about what they are dealing with. They are the ones living with the condition day in and day out. They know how they are feeling and what they are having to cope with. Do not just dismiss a symptom, as happened with us initially, with the choking. See if anyone else is reporting the same as I bet you will find they are. Work with the patient groups. They want to see success just as much as you. We are continuously teaching the medical professionals when we attend hospital appointments or get admitted to hospital. Even the specialists at our MDT clinics are learning from us each time we attend a clinic.

\footnotetext{
${ }^{1}$ Wolfram Syndrome UK, Worthing, West Sussex, UK
}

Correspondence: Tracy Lynch (info@pedres.org)

Received: 30 October 2019 Accepted: 30 October 2019

Published online: 7 November 2019 